\title{
I2MMS: An Interactive Multi Modal Visual Search Technique
}

\author{
Yojana S. Pawale ${ }^{1}$, P. R. Devale ${ }^{2}$ \\ Research Scholar, Dept. of Information Technology, B. V. D. U, College of Engineering, Pune City, India ${ }^{1}$ \\ Professor, Dept. of Information Technology, B. V. D. U., College of Engineering, Pune City, India ${ }^{2}$
}

\begin{abstract}
Now days, image searching technique is popularized on mobile phones where mobile users search and find desired images through their mobiles. But it is not easy to find desired image through mobile, because mobile phone' screen is too small to display full image. It is also difficult for users to provide input on mobile phones to find targeted image. Hence, by considering all these issues, we have developed one image searching technique called - I2MMS: An Interactive Multi Modal Visual Search Technique. In text based technique, if user provides lengthy queries to find desired image then it will be difficult for system to process that whole query and provides desired images to users and also not user friendly. Hence, we have proposed a new technique for image searching where system accepts multimodal input that can be voice, text and image. This system is useful in a case where users do not know exact name of an image but by describing it using either text or speech or by providing any other relevant image, users can easily find targeted image. The ANN technique is also added into it to increase the performance of the system. The system works in three phases- 1) Image Composition, 2) Image Processing, 3) ANN. Lastly, before providing images to users as an output, images are divided into two sets- positive and negative. All relevant images are present in positive set and non relevant images are present in negative set. Lastly images present in positive set are provided to the user as a final output. Hence our approach improves the quality of image searching technique and provides easy way to finds targeted image.
\end{abstract}

Keywords: Image Processing, Artificial Intelligence, Content Based Image Retrieval, Multimodal Input

\section{INTRODUCTION}

Image searching is a technique of searching, finding and find some more relevant images. Google Googgles[4], retrieving desired images from database. A large database Point and Find[5], Snaptell[6] uses this technique. Some is present at server site that contains no of images. The extra information of an output images is also provided by system accepts input query from user and provides a set of these applications. The limitation of these applications is number of relevant images to user according to user's that it provides searching mechanism only for landmarks, query. Currently, image searching technique is populated CD covers, Products etc. One technique is popularized on on mobile phones. Most of the time, image search is mobile phone is speech recognition technique. Apple performed by mobile users to find local information like Siri[3] is an application that uses this technique in which local maps. But it is not easy to search and finds targeted speech recognition and language understanding images through mobile phones. It may face many approaches are used to provides knowledge based problems. For example, Mobile phone's screen is too small searching mechanism. Recently one more technique is to display full length image. Hence, it may affects on developed for image searching is JIGSAW+ [1]. This presentation part of the system. Another drawback is - a system performs join image search by accepting input as way of providing input to the system. On computer text, image or speech. This technique is also popularized system, keyboards and mice are used to provide input mice on mobile phones but it does not consider user's intention but in case of mobile phones, input is provided by to be search. It missed an issue of artificial intelligence. cameras, GPS, microphones and multi touch screens. For example, if user gives text query as an 'Apple'; then Hence, it is very difficult to provide inputs to system and system does not focus on the point that whether user wants not user friendly as well as machine friendly. Many images on 'apple fruit' or 'apple company's products'. techniques are developed for image searching. The basic Hence by referring all these techniques, we have technique is developed for image searching is text-based developed one mechanism called I2MMS: An Interactive technique in which user gives text query to system and Multi Modal Visual Search Technique. The system is retrieves desired images. But the drawback of tit is that if multimodal where text based and image based techniques user gives lengthy query to find an image then that lengthy are combined with speech based technique to retrieve query is neither user friendly nor machine friendly because quality images by adding ANN mechanism in it. This the fact is that mobile users use only 2.6 terms on average mechanism provides multimodal approach where system for search [2], which can be hardly express their search accepts input as an image, text or speech. This system is intent. One more technique is developed for image mostly same as JIGSAW+ but also applies LAB and ANN searching is photo - to - search technique. In this technique to improve the performance of the system and technique, user provides a query to system as an image to provides faster searching mechanism to users. The system 
works in three phases as - 1) Image composition 2) Image processing 3) ANN. The plan for remaining sections is as follow- section 2 describes concept of proposed system. Section 3 represents system's architecture. Section 4 discussed detailed description of each module of proposed system. Section 5 shows experimental work of proposed system and lastly section 5 concluded this concept.

\section{PROPOSED SYSTEM}

The proposed system provides joint mechanism for image searching on mobile phones by combining text based and image based searching mechanisms with speech recognition techniques. The system is multimodal which accepts input as an either image or text or speech.

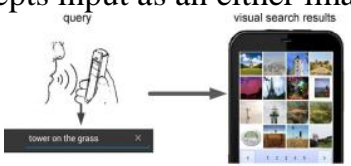

(a)

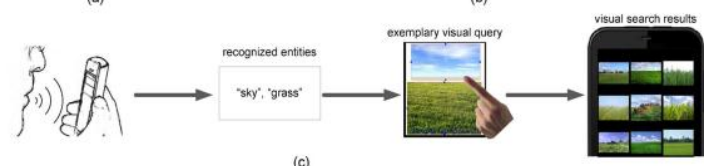

Fig.1. Three modes of mobile visual search: (a) voice/text-to-search, (b) photo-to-search, (c) our proposed visual search system.

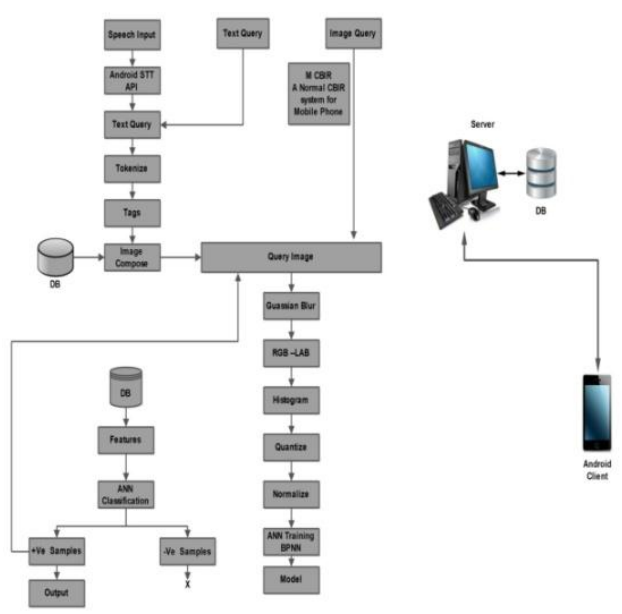

Fig.2 Architecture of our proposed system
In the last phase, the result of image processing is used to apply ANN mechanism on it. Here, BPNN mechanism is applied on received image for further processing. Then, system searches an image in their own database by taking ANN features of it and result of BPNN process of an input image and compares parameters of them and provides most relevant images to users. Before providing relevant images to user, two sets are created by the system-positive Above figure shows the basic idea of proposed system. and negative sets. Positive set contains number of relevant Fig. 1(a) represents voice/text-to-search technique in images and negative set contains number of non relevant which user provides input as either voice or text and images. Lastly, images present in the positive set are retrieves relevant images. Fig. 1(b) represents photo-to- provided to the user as a final output. Here, users can again search technique where user gives image query and select one output image and provides it as an input query retrieves more relevant images. Fig. 1(c) represents basic to the system to find more relevant images.

idea of proposed system. In proposed system, user A. Image Composition

provides input query to the system that can be either text In the first module, user provides input query as an either or voice or image and then according to that query, speech or text or image. If user provides speech query then composite images are provided to the users. After that system converts it into text. Android's STT API is used to User selects one image among them and retrieves more convert speech into text. Then, tokenization process is relevant images. For example, if user wants to search an applied on text query where text is decomposed into images for Punjabi restaurants in pune having red door. different tokens. Tokens are converted into tags and passes Then, system searches for composite images in their to the next phase. This step is performed because system database having all these entities as - Punjabi restaurants, searches images within database according to the tokens. pune and red door. Then user selects one composite image Hence, these tokens are separated by tags. After that, among them and retrieves more relevant images.

III. ARCHITECTURE OF PROPOSED SYSTEM The system works in three main phases - 1) Image Composition, 2) Image Processing, 2) ANN.

As shown in fig. 2, the system accepts input as either voice or text or image. If system accepts input as a voice then converts it into text and searches for no of relevant composed images within its own database and shown back to the user. Then user selects one composed image among them. After that system again accepts this image query as an input for further processing. User can directly provide image query at the first time for retrieving more relevant images. In the next phase image processing is performed on query image to extract features of it and provides to the next phase which requires for applying ANN technique. Before processing the query image, it converts into LAB form to improve the quality of an input image.

System searches for composed image in its own database according to the keywords and provides to the users. For example, if the text query is - "Restaurants in Pune". Then system accepts that text and converts it into keywords as'Restaurant' and 'Pune'. These tokens are separated by tags and related composed images are searched in system's database and provides to users. Here, System searches for composed images that having both the keywords i.e. 'Restaurant' and 'Pune'. User selects one composed image among them. Then, system accepts that composed image as an input query and passes it to next phase. It is also possible to provide input as an image at the first time to the system and finds more relevant images from system by performing next process.

\section{B. Image Processing}

This phase accepts input image and blurring it by applying Gaussian function. The image is converted into Gaussian form to reduce image noise. Then image is converted into 


\section{International Journal of Advanced Research in Computer and Communication Engineering}

Vol. 4, Issue 5, May2015

LAB form to improve the quality of an image. Here, $\mathrm{L}$ is the result of BPNN process and finds most relevant images stands for lightness and a, b are called color-opponent and provides to the user.

dimensions, based on nonlinearly compressed coordinates.

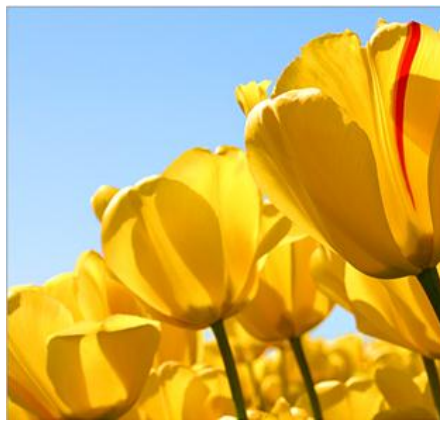

Fig. 3(a) Original image

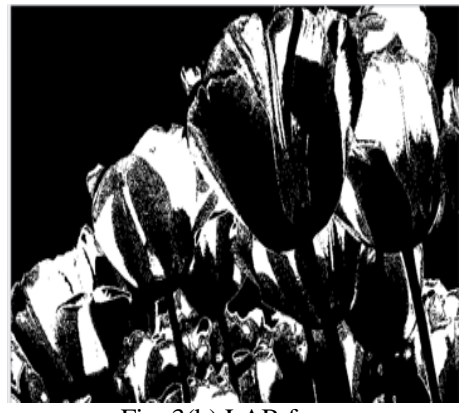

Fig. 3(b) LAB form

Fig. 3 shows LAB form of original image. LAB image is simply an image present in black and white form but in qualitative form. It helps to extract correct features of input image.

Then, histogram is created of LAB image. Histogram is a graphical representation of an image where horizontal axes represent tonal variation and vertical axes represent number of pixels in that particular tone. Histogram helps to judge the entire tonal distribution at a glance. Then, image is quantized after histogram is created. In the last step of image processing, image is normalized to apply ANN technique on it to provide more relevant images to the user.

\section{C. $A N N$}

This is the last phase of the system in which system accepts the result of image processing to extract required features of an image to apply ANN mechanism on it. This phase is called as a key part of the system because it focuses on the user's psychological factors behind searching. It concentrates on user's intention to be search that is what exactly user wants.

For example, if user gives a text query as a 'Head', then the point - whether user wants images related to 'Human head' or 'Head - of - Department' is considered here. For that BPNN mechanism is applied on image and then compares ANN parameters of an images present in their database with the parameters of query image taken form

\section{EXPERIMENTAL WORK}

Initially, we have provided one form to user in which user enters IP address of server to start an application. Then, on the next form, three options are shown to user to find relevant images as shown in fig. 5.

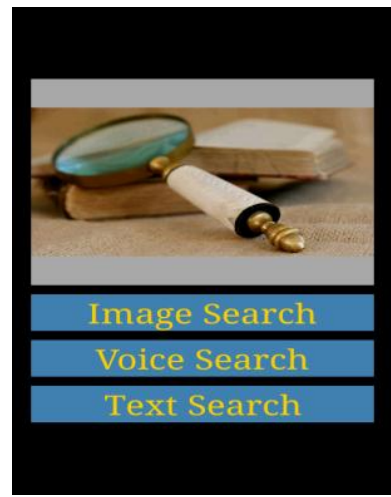

Fig. 5 Multimodal input

Depending on the user's choice, image is searched and retrieved from database. Fig. 6 is showing some result of our system. Here, user had provided text query to the system. Depending on that query some composed images are retrieved and shown to user as an output.

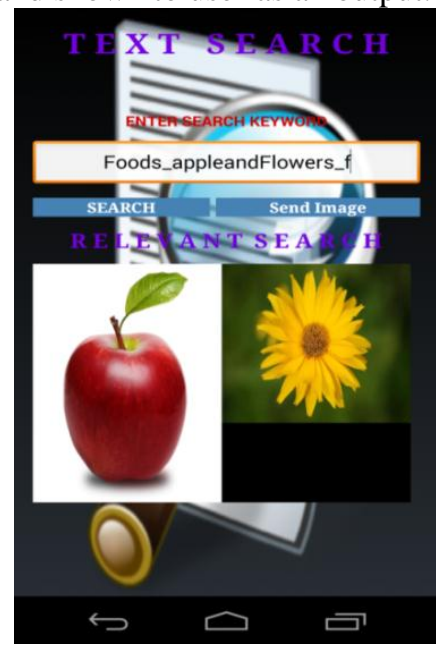

Fig. 6 Result of text query

If user selects one composed image among these two output images, For example, 'Apple' image. Then system accepts this apple image as an query and provides some more images related to an 'apple' image as shown in fig. 7 


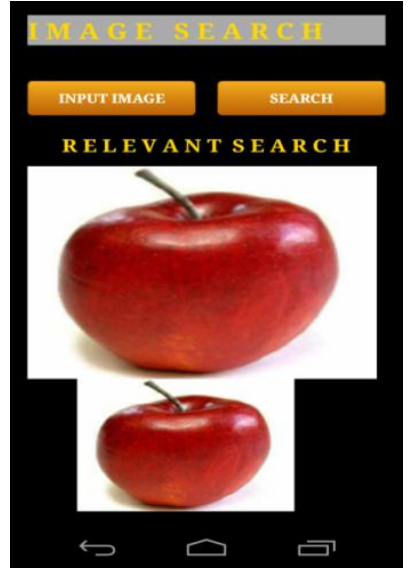

Fig. 7 Relevant images

V. CONCLUSION

The system provides powerful mechanism for image searching on mobile by providing multimodal approach. User can be able to provide any kind of input that is text, image and speech. The system concentrates on user's psychological factor behind image searching. This system works well in a case where user does not know exact name of an image then by describing it using text or speech, user can be able to get desired images. Lastly system separates number of images into two sets as positive and negative. Positive set represents relevant images and negative set represents non relevant images. Positive set is provided to user as a final output.

\section{REFERENCES}

[1] Houqiang Li, Senior Member, IEEE, Yang Wang, Tao Mei, Senior Member, IEEE, Jingdong Wang, Senior Member, IEEE, and Shipeng Li, Fellow, IEEE "Interactive Multimodal Visual Search on Mobile Device," IEEE TRANSACTIONS ON MULTIMEDIA, VOL. 15, NO. 3, APRIL 2013.

[2] K. Church, B. Smyth, P. Cotter, and K. Bradley, "Mobile information access: A study of emerging search behavior on the mobile internet," ACM Trans. Web, vol. 1, no. 1, May 2007.

[3] Siri. [Online]. Available: http://www.apple.com/iphone/features/siri.html/

[4] Google Goggles. [Online]. http://www.google.com/mobile/goggles/

[5] NOKIA Point and Find. [Online]. Available: http://pointandfind.nokia.com/

[6] SnapTell. [Online]. Available: http://www.snaptell.com/

[7] H. Bay, T. Tuytelaars, and L. Van Gool, "SURF: Speeded-up robust features," in Proc. ECCV, 2008, vol. 110, no. 3, pp. 346-359.

[8] E. Rublee, V. Rabaud, K. Konolige, and G. Bradski, "ORB: An efficient alternative to SIFT or SURF," in Proc. IEEE Int. Conf. Comput. Vis., Nov. 2011, pp. 2564-2571.

[9] V. Chandrasekhar, G. Takacs, D. Chen, S. Tsai, R. Grzeszczuk, and B. Girod, "CHoG: Compressed histogram of gradients a low bit-rate feature descriptor," in Proc. IEEE Conf. Comput. Vis. Pattern Recogn., Jun. 2009, pp. 2504-2511.

[10] M. Jia, X. Fan, X. Xie, M. Li, and W. Ma, "Photo-to-search: Using camera phones to inquire of the surrounding world," in Proc. Mobile Data Manag., 2006.

[11] S. Tsai, H. Chen, D. Chen, G. Schroth, R. Grzeszczuk, and B. Girod, "Mobile visual search on printed documents using text and low bitrate features," in Proc. IEEE Int. Conf. Image Process., Sep. 2011, pp. 2601-2604.

[12] V. Chandrasekhar, D. M. Chen, A. Lin, G. Takacs, S. S. Tsai, N. M. Cheung, Y. Reznik, R.Grzeszczuk, and B.Girod, "Comparison of local feature descriptors for mobile visual search," in Proc. IEEE Int. Conf. Image Process., 2010, pp. 3885-3888.

[13] X. Liu, Y. Lou, A.W. Yu, and B. Lang, "Search by mobile image based on visual and spatial consistency," in Proc. IEEE Int. Conf.Multimedia Expo, 2011, pp. 1-6.

Copyright to IJARCCE 